\title{
Survival probability in Generalized Rosenzweig-Porter random matrix ensemble.
}

\author{
G. De Tomasi ${ }^{1}$, M. Amini ${ }^{2}$, S. Bera ${ }^{3}$, I. M. Khaymovich ${ }^{1,4}$, V. E. Kravtsov ${ }^{5,6,7}$
}

1 Max-Planck-Institut für Physik komplexer Systeme, Nöthnitzer Straße 38, 01187 Dresden, Germany

2 Department of Physics, University of Isfahan(UI) - Hezar Jerib, 81746-73441, Isfahan, Iran 3 Department of Physics, Indian Institute of Technology Bombay, Mumbai 400076, India 4 Institute for Physics of Microstructures, Russian Academy of Sciences, 603950 Nizhny Novgorod, GSP-105, Russia

5 Abdus Salam International Center for Theoretical Physics - Strada Costiera 11, 34151 Trieste, Italy

6 L. D. Landau Institute for Theoretical Physics - Chernogolovka, Russia

7 Kavli Institute for Theoretical Physics, Kohn Hall, University of California, Santa Barbara, CA 93106-4030, U.S.A.

* detomasi@pks.mpg.de

July 14, 2022

\begin{abstract}
We study analytically and numerically the dynamics of the generalized RosenzweigPorter model, which is known to possess three distinct phases: ergodic, multifractal and localized phases. Our focus is on the survival probability $R(t)$, the probability of finding the initial state after time $t$. In particular, if the system is initially prepared in a highly-excited non-stationary state (wave packet) confined in space and containing a fixed fraction of all eigenstates, we show that $R(t)$ can be used as a dynamical indicator to distinguish these three phases. Three main aspects are identified in different phases. The ergodic phase is characterized by the standard power-law decay of $R(t)$ with periodic oscillations in time, surviving in the thermodynamic limit, with frequency equals to the energy bandwidth of the wave packet. In multifractal extended phase the survival probability shows an exponential decay but the decay rate vanishes in the thermodynamic limit in a non-trivial manner determined by the fractal dimension of wave functions. Localized phase is characterized by the saturation value of $R(t \rightarrow \infty)=k$, finite in the thermodynamic limit $N \rightarrow \infty$, which goes to $k=1$ as the initial state is taken to be localized to a single-site.
\end{abstract}

\section{Contents}

$\begin{array}{lll}1 & \text { Introduction } & 2\end{array}$

2 Survival probability in terms of eigenfunctions and eigenvalues 
3 Ansatz for eigenvectors of GRP model 5

4 The eigenvector overlap function $K(\omega)$. 6

$5 \quad R(t)$ and the eigenvalue distribution 7

6 Approximations for $R(t)$. 8

7 Oscillations in $R(t)$ and the spectral rigidity 9

8 Numerical test for ergodicity 12

9 Extracting fractal dimension $D$ from $R(t)$ in the NEE phase 13

10 Residual oscillations in $R(t)$ in the NEE phase 14

$11 R(t)$ in the localized phase $\quad 14$

12 Conclusions

13 Acknowledgements 16

References 17

14 Appendix 19

\section{Introduction}

Existence of a transition between a diffusive metal and a perfect insulator at a finite energy density in disordered interacting quantum systems isolated from the environment [1-3] has attracted a lot of attention and generated a rapidly expanding field of Many-Body Localization (MBL). However, the expected diffusive behavior of a normal metal in the delocalized phase of such systems has not been completely confirmed in several numerical simulations. Instead a sub-diffusive behavior has been often found [4 7] raising a question of an existence of the nonergodic extended (NEE) phase. Indeed, in such a phase, often nicknamed as "bad metal" [8,9, many-body wave functions are neither localized nor occupying all the available Hilbert space, but are like multifractal one-particle wave functions at the transition point of an ordinary Anderson localization problem [10]. The existence of NEE phase is of principle importance as it implies breakdown of conventional Boltzmann statistics. Moreover, the hierarchical, multifractal structure of eigenstates and hence of local spectrum (fractal mini-bands $[9]$ ) in interacting qubit systems is important for a rapidly developing part of computer science, the machine learning [11], as it allows for a continuous learning process with focusing on progressively more detailed properties of a learning subject. Nevertheless, the persistence of this NEE in the thermodynamic limit is still under debate, specifically, in recent works it has been argued that the NEE phase might only be transient, which implies that it crossover to 
a diffusive phase at long times in the thermodynamic limit 12,13. For this reason it is of fundamental importance to study a model in which this phase is rigorously proved to exist in order to give an efficient criteria to characterize it in generic cases.

One of the consequences of the lack of ergodicity in such a phase is the behavior of the survival probability $R(t)=|\langle\Psi(t) \mid \Psi(0)\rangle|^{2}$ at large times $t \rightarrow \infty$ in a system of finite dimension $N$ of the Hilbert space, with $|\Psi(0)\rangle$ being the initially prepared state,

$$
R(t \rightarrow \infty) \propto N^{-D}
$$

Here $D$ is the eigenfunction fractal dimension. Ergodicity implies an asymptotic equipartition over all available many-body states, and thus $D=1$, while in an MBL phase only a finite number of states are involved, and therefore $D=0$. The NEE phase is characterized by $0<D<1$, so that the volume in the Hilbert space occupied at $t \rightarrow \infty$ is infinite $N^{D} \rightarrow \infty$ in the thermodynamic limit, yet it constitutes zero fraction $N^{D-1} \rightarrow 0$ of all states. The survival probability $R(t)$ is of particular importance for many-body spin systems, as it is a proxy of the spin autocorrelation function $\langle\sigma(0) \sigma(t)\rangle$ in interacting spin-qubit systems [20].

A simple single-particle system where all three phases, ergodic, localized and NEE, exist in the corresponding range of parameters, is the Generalized Rosenzweig-Porter (GRP) random matrix ensemble 14, 15] 1. The GRP ensemble 15 of random real matrices is characterized by independent identically distributed entries $H_{n m}$, such that:

$$
\overline{H_{n m}}=0, \quad \overline{\left(H_{n n}\right)^{2}}=1, \quad \overline{\left(H_{n \neq m}\right)^{2}}=\frac{\lambda^{2}}{N^{\gamma}},
$$

where $\bar{A}$ denotes ensemble average of $A, N$ is the matrix size, and $\gamma, \lambda$ are real parameters. The existence of the NEE phase and a transition to the ergodic state in this model has been recently suggested in Ref. [15], and further confirmed in Ref. [20, 21], culminating with a rigorous proof in Ref. [16. In particular, at $\gamma<1$ the system is fully ergodic $(D=1)$ and behaves as the Gaussian ensemble [22], at $1<\gamma<2$ the NEE phase is realized with $D=2-\gamma$ and for $\gamma>2$ the system is localized $(D=0)$. The critical points

$$
\gamma_{E}=1, \quad \gamma_{c}=2
$$

indicate the transitions from the NEE phase to ergodic (ergodic transition) and localized phases (localization transition), respectively. In this paper we consider the time dependence of the survival probability

$$
R(t)=\overline{\left|\left\langle\Psi_{0}\left|\widehat{P}_{f} e^{-i t \hat{H}} \widehat{P}_{f}\right| \Psi_{0}\right\rangle\right|^{2}}
$$

of the initial state $|\Psi(0)\rangle=\widehat{P}_{f}\left|\Psi_{0}\right\rangle$, being a single-particle analogue of a product state $\left|\Psi_{0}\right\rangle$ represented by the basis vector in which only one component is 1 and all the others are equal to 0 , projected by $\widehat{P}_{f}=\sum_{n=N / 2(1-f)}^{N / 2(1+f)}\left|\psi_{n}\right\rangle\left\langle\psi_{n}\right|$ to a finite small fraction $0<f \ll 1$ of eigenstates $\left\{\left|\psi_{n}\right\rangle\right\}$ of $\widehat{H}$ in the middle of the spectrum. The final state $\Psi(t \rightarrow \infty)$ is a random $N$-vector in which $N^{D}$ elements are of the order of $\sim N^{-D}$ and all the others are much smaller. The main reason to use the projector is that $\widehat{P}_{f}$ provides an opportunity to use $R(t)$ as a probe to

\footnotetext{
${ }^{1}$ Recently, the presence of a whole NEE phase has been found in several static 17,18 and periodicallydriven 19 long-range random matrix models from a different universality class.
} 
characterize the ergodic phase. On the other hand, it is helpful for numerical reasons, as it reduces finite size effects. Indeed, usually the finite size corrections to the fractal dimension depend on the energy $(D=D(E, N))$, thus projecting to a small fraction of eigenstates $f \ll 1$ one can neglect these variations in $D$ versus $N$, making the data analysis easier.
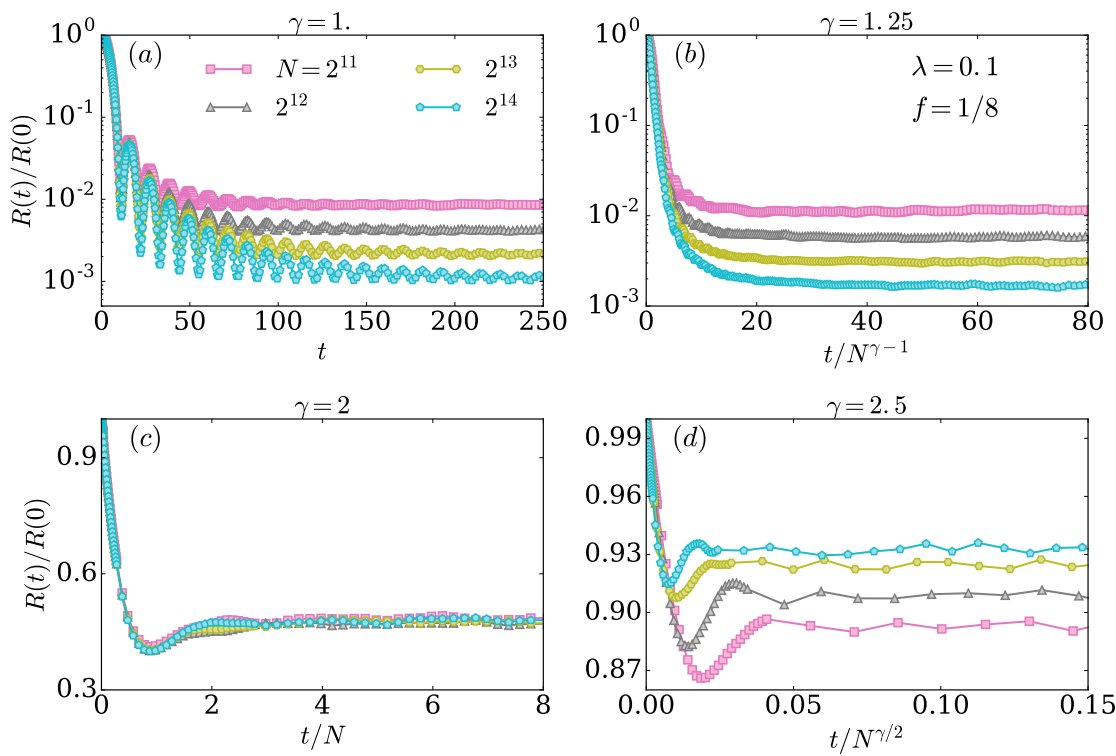

Figure 1: (Color online) Evolution of $R(t)$ with increasing $\gamma$ for the box distribution of onsite disorder. The ergodic transition is marked by the onset of oscillations in $R(t)$ which survive the thermodynamic limit $N \rightarrow \infty$. The value of $R(\infty) \propto N^{-D}$ decreases with increasing the system size for $\gamma<2$ and it increases and approaches its initial value $R(0)$ in the thermodynamic limit for $\gamma \geq 2$. At the localization transition $\gamma=2$ the form of $R(t)$ is scale-invariant. The numerical data shown in the paper is averaged over $N_{r}=1000$ disorder realizations and $N_{x}=16$ basis states $\left|\Psi_{0}\right\rangle, f=1 / 8, \lambda=0.1$.

The main results of this paper can be formulated as follows (see also Fig. 1):

- In the ergodic phase $\gamma<1$ the survival probability, Eq. (4), exhibits pronounced oscillations in time. The amplitude of these oscillations decays as $\propto t^{-2}$ with time and the frequency of the oscillations is given by the spectral width of the initially prepared wave packet and thus is inversely proportional to $f$. The presence of such oscillations and the character of their decay with time in the thermodynamic limit $N \rightarrow \infty$ in general depends on the initial spectral decomposition of the prepared wave packet [23, 25 27$]$ and in the case of the projected basis state, Eq. (4) acquires a standard form given by $R(t) \sim[\sin (\pi t / \Delta t) /(\pi t / \Delta t)]^{2}$, where $\Delta t \propto f^{-1}$. Moreover, it can be used as a probe of the rigidity of the energy levels of the system, which is established in the fully ergodic phase.

- In the NEE phase the survival probability decays exponentially $R(t)=\exp [-\Gamma(N) t]$ with time as long as $R(t) \gg N^{-D}$. The characteristic decay rate $\Gamma(N) \propto N^{D-1}$ is determined by the fractal dimension $D$. In this phase, some oscillations in $R(t)$ may exist, but only as a finite-size effect. 
- In the localized phase $R(t)$ tends to a constant $R(\infty)$ as $t \rightarrow \infty$, where $R(\infty)$ remains finite and tends to its initial value $R(0)$ as $N \rightarrow \infty$.

\section{Survival probability in terms of eigenfunctions and eigen- values}

The survival probability $R(t)$ can be expressed in terms of eigenvalues $\varepsilon_{n}$ and eigenfunctions $\psi_{n}(r)$ of the state $n$ as:

$$
R(t)=\sum_{n, m}^{\prime} \overline{\left|\psi_{n}(0)\right|^{2}\left|\psi_{m}(0)\right|^{2} \cos \left[\left(\varepsilon_{n}-\varepsilon_{m}\right) t\right]}
$$

where sums run over eigenstates indices, which belong to the projected subspace $\sum_{n}{ }^{\prime}=$ $\sum_{n \in(1-f) N / 2}^{(1+f) N / 2}$ containing a finite fraction $f$ of the system spectrum 2 , Indeed, the initial basis state $\left\langle r \mid \Psi_{0}\right\rangle \equiv \Psi_{0}(r, t=0)=\delta_{r, 0}$ which is non-zero only on one site $r=0$, can be represented as follows using the completeness of the set of eigenfunctions:

$$
\Psi(r, t=0)=\sum_{n} \psi_{n}^{*}(0) \psi_{n}(r)
$$

The application of the projector $\widehat{P}_{f}$ to this state to form the initial wave function $\Psi_{f}(r, t=$ $0) \equiv\left\langle r \mid \widehat{P}_{f} \Psi_{0}\right\rangle$ implies restriction in the summation to $\sum_{n}{ }^{\prime}$. The Schrödinger dynamics $\psi_{n}(r, t)=\psi_{n} e^{i \varepsilon_{n} t}$ then leads to:

$$
\Psi_{f}(r, t)=\sum_{n}^{\prime} \psi_{n}^{*}(0) \psi_{n}(r) e^{i \varepsilon_{n} t}
$$

which immediately results in Eq. $\sqrt[5]{5}$ for $R(t)=\overline{\left|\Psi_{f}(0, t)\right|^{2}}$.

\section{$3 \quad$ Ansatz for eigenvectors of GRP model}

The key point of our analytical consideration is the following ansatz (see also [18,21,28]) for the amplitude of an eigenstate of GRP model, $\psi_{n}(r)$ at site $r$, with its maximum occurring at $r=n$. This ansatz works for all three phases [18,21]:

$$
\left|\psi_{n}(r)\right|^{2}=\frac{\left|H_{n r}\right|^{2}}{\left(\varepsilon_{n}-\varepsilon_{r}\right)^{2}+\Gamma(N)^{2}} .
$$

For NEE states $(1<\gamma<2)[15$ the characteristic energy

$$
\Gamma(N) \propto N^{D} \delta_{m b} \sim N^{-(\gamma-1)} \propto N^{D-1} .
$$

has a meaning of the width of a mini-band [9, 29] in the local spectrum formed by $N^{D}$ states sharing the same fractal support set in the coordinate space. On this set of sites the

\footnotetext{
${ }^{2}$ The choice of the initial site $r=0$ is irrelevant after taking disorder average.
} 
eigenfunction coefficients of states belonging to a mini-band are large $\sim N^{-D}$, while outside of it they are much smaller $\sim N^{2-D}$. These states are separated by the typical level spacing $\delta_{m b}$ of the order of the global one $\delta \sim N^{-1}$. The entire coordinate space consists of $N^{1-D}$ non-intersecting fractal support sets each one corresponding to a mini-band in the global spectrum. Thus the entire global spectrum is a unification of $N^{1-D}$ mini-bands of which typically only one is seen in the local spectrum (e.g. in the LDoS). This picture is encoded in Eq. (8). Indeed, the typical energy difference $\left|\varepsilon_{n}-\varepsilon_{r}\right| \sim 1$ according to Eq. (8) corresponds to the typical amplitude $\left|\psi_{n}(r)\right|_{\text {typ }}^{2} \sim\left|H_{n m}\right|^{2} \propto N^{-\gamma}$, while the amplitude of states inside the mini-band $\left|\varepsilon_{n}-\varepsilon_{r}\right|<\Gamma(N)$ is much larger $\left|\psi_{n}(r)\right|^{2} \sim\left|H_{n m}\right|^{2} /[\Gamma(N)]^{2} \propto N^{-(2-\gamma)}=N^{-D}$.

For ergodic states $\gamma<1$ the global density of states (DoS) has a semi-ellipse form [15,30]:

$$
\rho(\varepsilon)=\frac{\sqrt{2 S-\varepsilon^{2}}}{\pi S}, \quad S=\sum_{n}\left\langle\left|H_{m n}\right|^{2}\right\rangle=f \lambda^{2} N^{1-\gamma},
$$

so that in the center of the band $\delta(N)=(\rho(0) N)^{-1} \propto N^{-(1+\gamma) / 2}$ [15. The mini-bands are absent in this case, and

$$
\Gamma(N)=\delta(N) N \propto N^{(1-\gamma) / 2},
$$

grows with $N$ like the global band-width $\sim N \delta$. In this case Eq. (8) gives the typical and the maximal amplitudes of the same order $\left|\psi_{n}(r)\right|^{2} \sim\left|H_{n r}\right|^{2} /[\Gamma(N)]^{2} \sim N^{-1}$.

For localized states $(\gamma>2)$,

$$
\Gamma(N)=\lambda N^{-\gamma / 2} \ll \delta,
$$

is just the mean splitting $\left(\overline{\left|H_{n m}\right|^{2}}\right)^{1 / 2}$ of two resonance levels 15. In this case the peak amplitude at $n=r$ is

$$
\left|\psi_{n}(n)\right|^{2}=1+O\left(N^{-(\gamma-2)}\right)
$$

the maximal $\left|\psi_{n}(r)\right|^{2}$ of a typical wave function at $r \neq n$ according to Eq. (8) is of the order of:

$$
\left|\psi_{n}(r)\right|_{\max }^{2}=\left|H_{n r}\right|^{2} /\left[\delta^{2}+\Gamma^{2}\right] \sim\left|H_{n r}\right|^{2} / \delta^{2} \sim N^{-(\gamma-2)} \ll 1
$$

while the typical

$$
\left|\psi_{n}(r)\right|_{\text {typ }}^{2}=\exp \left[\overline{\ln |\psi|^{2}}\right] \sim\left|H_{n r}\right|^{2} /\left[1+\Gamma^{2}\right] \sim\left|H_{n r}\right|^{2} \sim N^{-\gamma},
$$

scales in the same way with $\gamma$ as in NEE phase [15. Equation (13) expresses an important property of GRP model that the wave function is essentially localized on one single site, i.e. the localization radius is equal to zero.

\section{The eigenvector overlap function $K(\omega)$.}

An important measure of the wave function statistics is the overlap function 29, 31]

$$
K\left(\varepsilon_{n}-\varepsilon_{m}\right)=N \sum_{r} \overline{\left|\psi_{n}(r)\right|^{2}\left|\psi_{m}(r)\right|^{2}}{ }_{\text {off }}
$$

it enters matrix elements of all the local interactions. In particular, it is responsible for the enhancement of superconducting transition temperature in dirty metals close to metalinsulator transition [32,33] and to recently suggested enhancement of phonon relaxation rate 
and electron-phonon cooling close to the localization transition [34]. Furthermore, $K(\omega)$ is the Fourier-transform of the survival probability which is an important dynamical measure relevant also for the many-body localization 24,27. Here $\bar{A}_{\text {off }}$ denotes averaging of $A$ over the off-diagonal matrix elements $H_{n m}$ only. Plugging Eq. (8) into Eq. (16) one obtains for delocalized phase in the limit $\Gamma(N) \gg \delta(N)$ :

$$
\begin{aligned}
& K\left(\varepsilon_{n}-\varepsilon_{m}\right)=2 \pi \rho(0) \frac{\lambda^{4}}{\Gamma} N^{2(1-\gamma)} \frac{1}{\left(\varepsilon_{n}-\varepsilon_{m}\right)^{2}+4 \Gamma^{2}}, \quad(n \neq m), \\
& K(0)=N I_{2} \equiv N \sum_{r} \frac{\left|\psi_{n}(r)\right|^{4}}{\left.\mid \varepsilon_{n}-\varepsilon_{m} \rightarrow 0\right) .}
\end{aligned}
$$

In deriving this equation we assumed that the eigenvalue fluctuations are independent from those of the off-diagonal matrix elements $H_{n m}$ [18, 20]. Then we switched from summation over $r$ to integration over $\varepsilon_{r}$ and used the fact that the convolution of two Cauchy functions is also the Cauchy function with the double width. This assumption is based on a large number of different paths contributing to a given quantum state which can be traced back to the infinite connectivity of each site. At such conditions the effect of one single off-diagonal matrix element to the eigenvalue is negligible.

In the localized phase, where $\Gamma(N) \ll \delta(N)$, the main contribution to Eq. $(16)$ is done by the two terms with $r=n$ and $r=m$ ? Taking into account that $\left|\psi_{n}(n)\right|^{2} \approx 1$, one obtains:

$$
\begin{aligned}
& K\left(\varepsilon_{n}-\varepsilon_{m}\right)=\frac{2 N^{1-\gamma}}{\left(\varepsilon_{n}-\varepsilon_{m}\right)^{2}+\Gamma^{2}}, \\
& K(0)=\frac{N^{1-\gamma}}{\Gamma^{2}}
\end{aligned}
$$

\section{$5 \quad R(t)$ and the eigenvalue distribution}

Finally we obtain the expression for $R(t)$ in terms of $K\left(\varepsilon_{n}-\varepsilon_{m}\right)$ :

$$
R(t)=f I_{2}+\frac{1}{N^{2}} \sum_{\substack{n, m \\ n \neq m}}^{\prime} \overline{K\left(\varepsilon_{n}-\varepsilon_{m}\right) \cos \left[\left(\varepsilon_{n}-\varepsilon_{m}\right) t\right]},
$$

where $\bar{A}_{\varepsilon}$ denotes averaging of $A$ over the eigenvalue distribution. In this equation $f$ is the fraction of all the states involved in the projection procedure in Eq. (4).

Eq. (19) can be rewritten

$$
R(t)=f I_{2}+\int d \varepsilon d \varepsilon^{\prime} C\left(\varepsilon, \varepsilon^{\prime}\right) K\left(\varepsilon-\varepsilon^{\prime}\right) \cos \left[\left(\varepsilon-\varepsilon^{\prime}\right) t\right]
$$

using the DoS correlation function:

$$
C\left(\varepsilon, \varepsilon^{\prime}\right)=\overline{\rho(\varepsilon) \rho\left(\varepsilon^{\prime}\right)}, \quad \rho(\varepsilon)=\frac{1}{N} \sum_{n}^{\prime} \delta\left(\varepsilon-\varepsilon_{n}\right) .
$$

\footnotetext{
${ }^{3}$ In the delocalized phases, $\Gamma(N) \gg \delta(N)$, the contribution of $r=n$ and $r=m$ are also of the same order as $K(\omega)$ itself.
} 


\section{Approximations for $R(t)$.}

In the absence of the exact solution for the joint eigenvalue-eigenvector statistics we start with simple approximations for the eigenvalue correlations. Let us first assume that the energy levels are uncorrelated $C\left(\varepsilon, \varepsilon^{\prime}\right)=\bar{\rho}(\varepsilon) \bar{\rho}\left(\varepsilon^{\prime}\right)$. As will be shown in Sec. 7 this assumption is valid at energy separations $\left|\varepsilon-\varepsilon^{\prime}\right| \gg \delta$ much greater than $\Gamma(N) / \ln N^{1-D}$ or times much shorter than $t_{E}=\Gamma^{-1} \ln N^{1-D}$. This time has a meaning of the Ehrenfest time in the chaotic dynamics. Then close to $\varepsilon=0$ we obtain from Eq. (20):

$$
R(t)=f I_{2}+C \int_{-\infty}^{\infty} d \omega K(\omega) \cos [(\omega t)], \quad C=\int[\rho(\varepsilon)]^{2} d \varepsilon
$$

This immediately results in an exponential behavior for in the NEE phase:

$$
R(t) \propto e^{-2 \Gamma t},\left(t<t_{E}\right),
$$

with a finite-size saturation value $R(\infty)=f I_{2} \propto N^{-D}$ at $t>t_{H}=1 / \delta(N)$, where $t_{H}$ is the Heisenberg time.

Another simple approximation is an assumption of absolutely rigid energy spectrum $\varepsilon_{n}-$ $\varepsilon_{n+\ell}=\ell \delta$. The validity of this approximation will be discussed later in Sec. 7. In this case it is convenient to use Eq. (19) and do summation first over $(n+m) / 2$ and then over $\ell=n-m$. We obtain:

$$
\begin{aligned}
& R(t)=f I_{2}+\frac{2}{N^{2}} \sum_{\ell=1}^{f N}(f N-\ell) K(\ell \delta) \cos (t \delta \ell)= \\
& f I_{2}-(2 f / N) K(\omega \rightarrow 0)+\frac{1}{N^{2}} \sum_{\ell=-f N}^{f N}(f N-\ell) K(\ell \delta) \cos (t \delta \ell) .
\end{aligned}
$$

The sum in Eq. 24) can be evaluated using the Poisson summation formula (see Appendix). The result at $t \ll 2 \pi / \delta$ is:

$$
R(t)-R(\infty)=\frac{A}{g} Y\left(\frac{t \Gamma}{\pi}, c\right)
$$

where

$$
c=\frac{f N \delta(N)}{2 \Gamma(N)}
$$

$A=2 \pi f \bar{\rho}(0)^{2} \lambda^{4} N^{2(1-\gamma)} /(\Gamma \delta), \bar{\rho}(0)$ is the mean DoS and $g=2 \Gamma / \delta$.

In the limit $2 \pi k \sqrt{c+1} \gg 1$ the function $Y(k, c)$ is very well approximated (see Fig. 2) by:

$$
Y(k, c) \approx \pi e^{-2 \pi|k|}+\frac{1}{2 c(\pi k)^{2}}-\frac{\cos (2 \pi k c)}{2(\pi k)^{2} c\left(c^{2}+1\right)} .
$$

Thus we conclude from Eqs. 27, 25) that the r.h.s. of Eq. 24 oscillates with the period inversely proportional to the band-width:

$$
\Delta t=\frac{2 \pi}{f N \delta}=\frac{2 \pi \bar{\rho}(0)}{f} .
$$



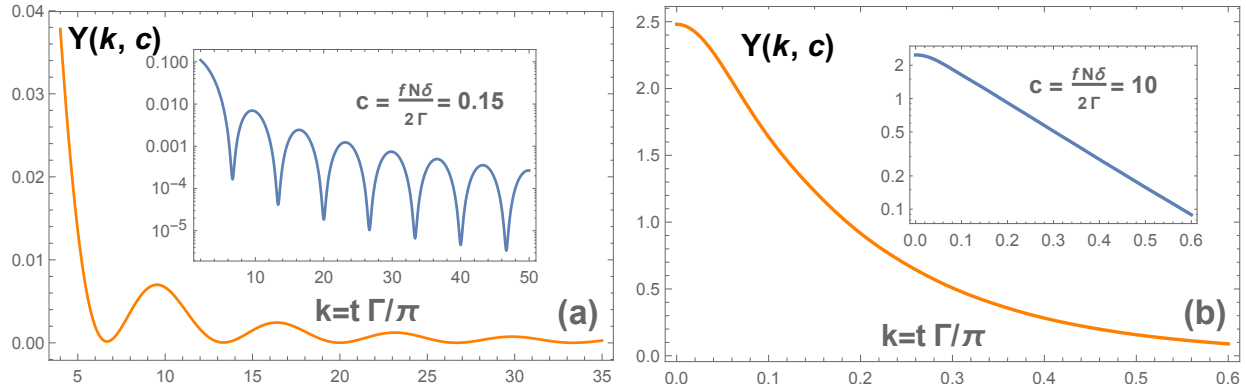

Figure 2: (Color online) $Y(k, c)$ (a) from Eq. (27) for $c=0.15$ and (b) from Eq. (27) for $c=10$. Insets show the same function in log-linear scale. In the ergodic phase $c \simeq f \ll 1$ is $N$ independent and small at small $f$. In the non-ergodic extended and localized phases $c \propto N^{\gamma-1}$ and $c \propto N^{\gamma / 2}$, respectively. It increases with increasing $N$, and oscillations disappear in the thermodynamic limit.

This period is $N$-independent for $\gamma>1$ and it is proportional to $N^{-(1-\gamma) / 2}$ for $\gamma<1$.

For ergodic phase, $\gamma<1$, in agreement with Eqs. (27) at $c \ll 1$ and Eq. (25) the survival probability, Eq. (24),

$$
R(t)-R(\infty)=R(0)\left[\frac{\sin (\pi t / \Delta t)}{\pi t / \Delta t}\right]^{2}
$$

coincides with the Fourier transform of the box-shaped DoS of states involved in the projection procedure in Eq. (4) and it oscillates as a function of $t$ and the amplitude of oscillations decays as $1 / t^{2}$ (see Fig. 2(a)). These oscillations are standard for fully ergodic phases, behaving as Gaussian ensembles [22] and have the same origin determined by the rigidity of the eigenspectrum as those considered in 23, 25, 26]. In our case because of the box-shaped DoS at $f \ll 1$ the oscillations decay as $t^{-2} \sqrt[4]{ }$, and not as $t^{-3}$ as in the case of a semi-circular DoS of Gaussian random matrix ensembles [25, 26] 5

For NEE phase, $1<\gamma<2$, corresponding to $c \sim N^{\gamma-1} \gg 1$ in Eq. (27), oscillations are suppressed in the thermodynamic limit.

Note that $A / g \sim 1 / f$ in Eq. (25) is an $N$-independent constant for extended states $(0<$ $\gamma<2)$ and $A / g \propto N^{1-\gamma / 2}$ vanishes in the thermodynamic limit for localized states $(\gamma>2)$. In the latter case $R(t) \equiv R(\infty)$ in the limit $N \rightarrow \infty$.

\section{Oscillations in $R(t)$ and the spectral rigidity}

As clearly observed in Fig. 3 the return probability $R(t)$, calculated using exact numerical simulations (see next section for details), shows oscillations in time as predicted with our previous approximations. The physical reason for these oscillations in $R(t)$ is the box-shaped DoS involved in the initial distribution of $\left|\Psi_{f}(r, t=0)\right|^{2}$ and the complete rigidity of the spectrum $\varepsilon_{\ell}=\ell \delta$. For random Hamiltonians the spectrum is also random but exhibits the Wigner-Dyson spectral rigidity in the ergodic phase. It implies the variance $\operatorname{var}(n)$ of the

\footnotetext{
${ }^{4}$ This $t^{-2}$ decay is like in realistic lattice many-body quantum systems $23.25 \mid 26$

${ }^{5}$ Another advantage of considering $f \ll 1$ is that it allows to use fast algorithms at large- $N$ numerical simulations aimed to identify the ergodic phase and the ergodic transition.
} 
number of levels in an energy interval containing $n$ levels on average,

$$
\operatorname{var}_{\operatorname{erg}}(n) \propto \ln (n), \quad n \gg 1,
$$

is small compared to statistically independent (Poissonian) fluctuation of levels, typical for localized states:

$$
\operatorname{var}_{\text {loc }}(n)=n
$$

This level rigidity ensures that the behavior of $R(t)$ can be approximated using a complete regular spectrum as done before. Indeed, the fluctuation of the width of the energy strip containing $\ell$ levels results in smearing of the edge $x= \pm f N$ of the spectrum by $w \sim \delta \sqrt{\ln (\ell)}$ (see Eq. (36) in Appendix). This makes the amplitude of oscillations acquiring an exponentially decaying factor $\exp (-w t)$ in addition to a power-law $t^{-2}$ decay due to edge singularity. However, the oscillations will not be affected by this exponential decay in the time interval $\Delta t \ll t \ll w^{-1}$. The existence of such an interval is guaranteed in the ergodic phase, as it requires only a condition:

$$
\frac{f N}{\ln ^{\frac{1}{2}}(\ell)} \gg 1, \quad \ell \leq \ell_{\text {edge }}=f N
$$

Moreover, even the Poisson level statistics by itself, Eq. (31), does not kill the oscillations, provided that $\Gamma \gg w \sim \delta \sqrt{\ell_{\text {edge }}} \sim N^{-\frac{1}{2}} 6$. In this case the condition similar to Eq. 32 reads:

$$
\sqrt{f N} \gg 1
$$

which is easily fulfilled. Much stronger effect on oscillations comes from eigenvector fluctuations reducing the jump size in $K(\omega)$ rather than from the smearing of this jump (see Eq. (36) in Appendix). This effect is quantified by a large value of the parameter $c$ in Eqs. (25, 26) when $N \delta \sim 1 / \bar{\rho}(0) \sim 1$ and $\Gamma \sim N^{-(1-\gamma)} \ll 1$.

In Fig. 2 we plot the function $Y(k, c)$ which determines $R(t)-R(\infty)$ via Eq. (25) in two cases: (a) small value of $c$ typical for the ergodic phase at a small fraction $f \ll 1$ of states considered and (b) for large value of $c$ typical for the NEE phase at large enough system size $N$. One can see that oscillations disappear at large $c$, since the main contribution to Eq. (27) at $t \Gamma \lesssim \ln c \sim \ln N^{1-D}$ is given by the first, exponentially decreasing term. Thus the region of validity of Eq. (23) is

$$
t<t_{E}=\Gamma^{-1} \ln N^{1-D} .
$$

Note that, unlike the fully ergodic phase (equivalent to Wigner-Dyson Gaussian ensembles) or diffusive systems with isotropic scattering, in GRP model the Ehrenfest time $t_{E}$, below which $R(t)$ decays exponentially, is parametrically large $t_{E} \sim \Gamma^{-1} \sim N^{1-D}$, though it is still smaller than the Heisenberg time $t_{H} \sim 1 / \delta \sim N$, at which the survival probability reaches its saturation. In between of $t_{E}$ and $t_{H}$ there is a complex revival behavior of $R(t)$ referred in the literature as a "correlation gap" 23,25,27.

Another important physical quantity which quantifies the spectral rigidity is the probability density $P(s, n)$ to have energy difference $\Delta E=s \delta(N)$ between two levels, provided that in between of them there are exactly $n-1$ other levels. Thus $P(s, 1)=P(s)$ is the well-known spacing distribution function, decaying exponentially for the Poisson level statistics $P(s)=e^{-s}$ and showing the level repulsion $P(0)=0$ for the Wigner-Dyson ensembles

\footnotetext{
${ }^{6}$ i.e., for $1<\gamma<3 / 2$. However for $\gamma>3 / 2$ the oscillations are completely absent.
} 

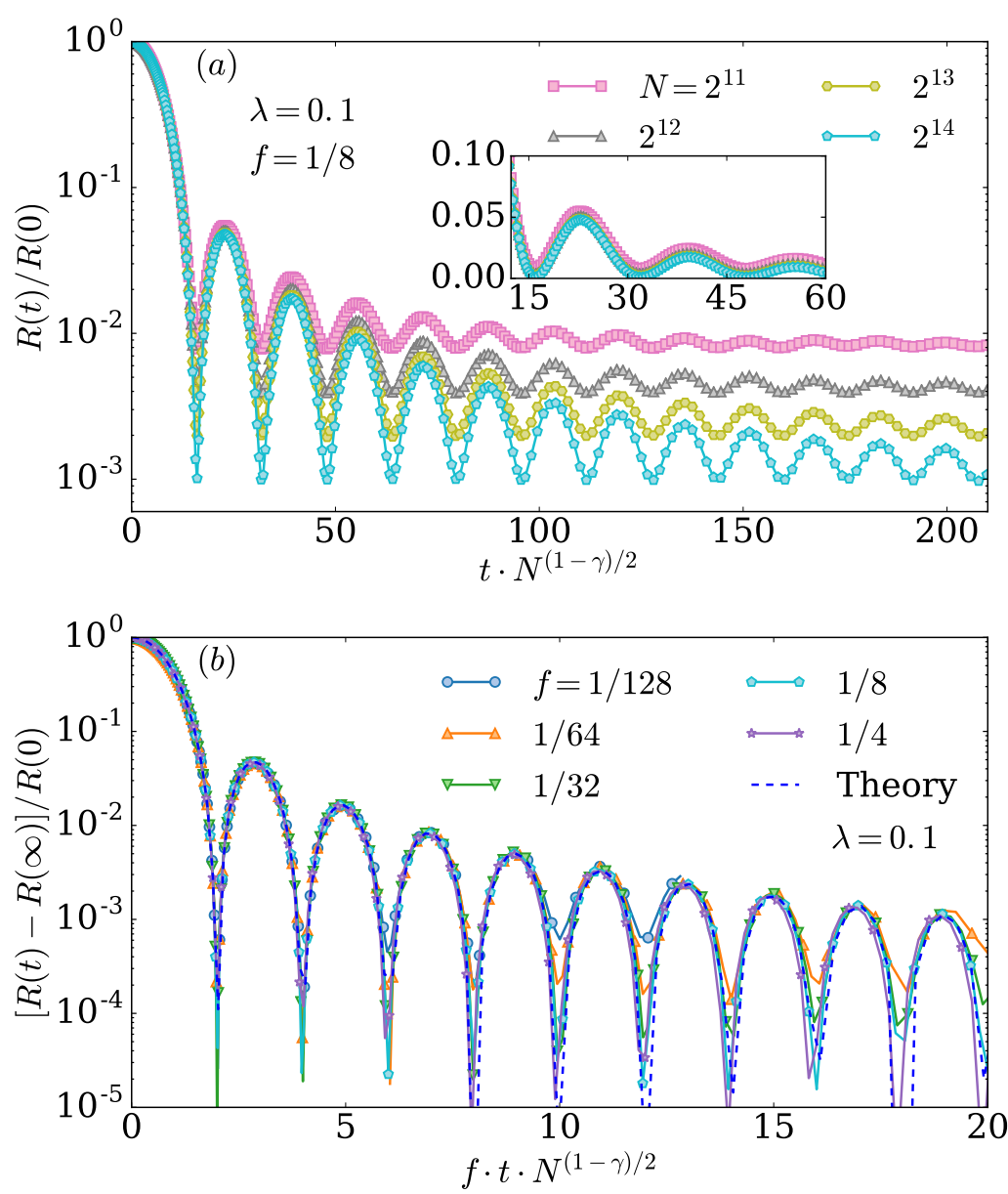

Figure 3: (Color online) Oscillations of survival probability $R(t)$ in the ergodic phase, $\gamma=0.25$ (a) for different matrix sizes $N$ and (b) for different fraction of states $f$ involved. The period of oscillations scales as $N^{-(1-\gamma) / 2}$, in accordance with Eq. (28). In the ergodic phase $c \ll 1$ the form of oscillations is well approximated (see Eqs. (25, 27)) by Eq. (29) shown as the dashed blue line.

$P(s)=(\pi s / 2) e^{-\pi s^{2} / 4}$. In Fig. 4 we plot the results obtained by exact diagonalization of GRP model for $P(s, n)$ statistics. The upper panel demonstrates that in the ergodic phase $P(s, n)$ has a Gaussian form $\ln P(s, n)=-(s-n)^{2} / \operatorname{var}(n)$, where $\operatorname{var}(n) \sim \ln (n)$, as it should be for the Wigner-Dyson level statistics.

In the NEE phase the character of $P(s, n)$ changes drastically. First, $P(s, n)$ for $s>n$ has a quasi Poisson tail $\ln P(s, n) \sim-(s-n) / \operatorname{var}_{R}(n)$ with $\operatorname{var}_{R}(n)=\chi n,(0<\chi<1)$ predicted long ago [35 for $P(s)$ at the Anderson transition point. However, for $n \gg 1$ it makes sense also to study $P(s, n)$ for $s<n$ and $|s-n| \ll n$ (lower-panel in Fig. 4). A remarkable fact is that the curves for $P(s, n)$ for $s<n$ and different $n$ collapse with the variance $\operatorname{var}_{L}(n) \sim n^{\beta}$, $\beta=1.6>1$. Such a super-Poisson behavior of the variance implies clustering which is related with the mini-band structure [9] where the level correlations inside a cluster of levels forming a mini-band are much stronger than between the mini-bands. 

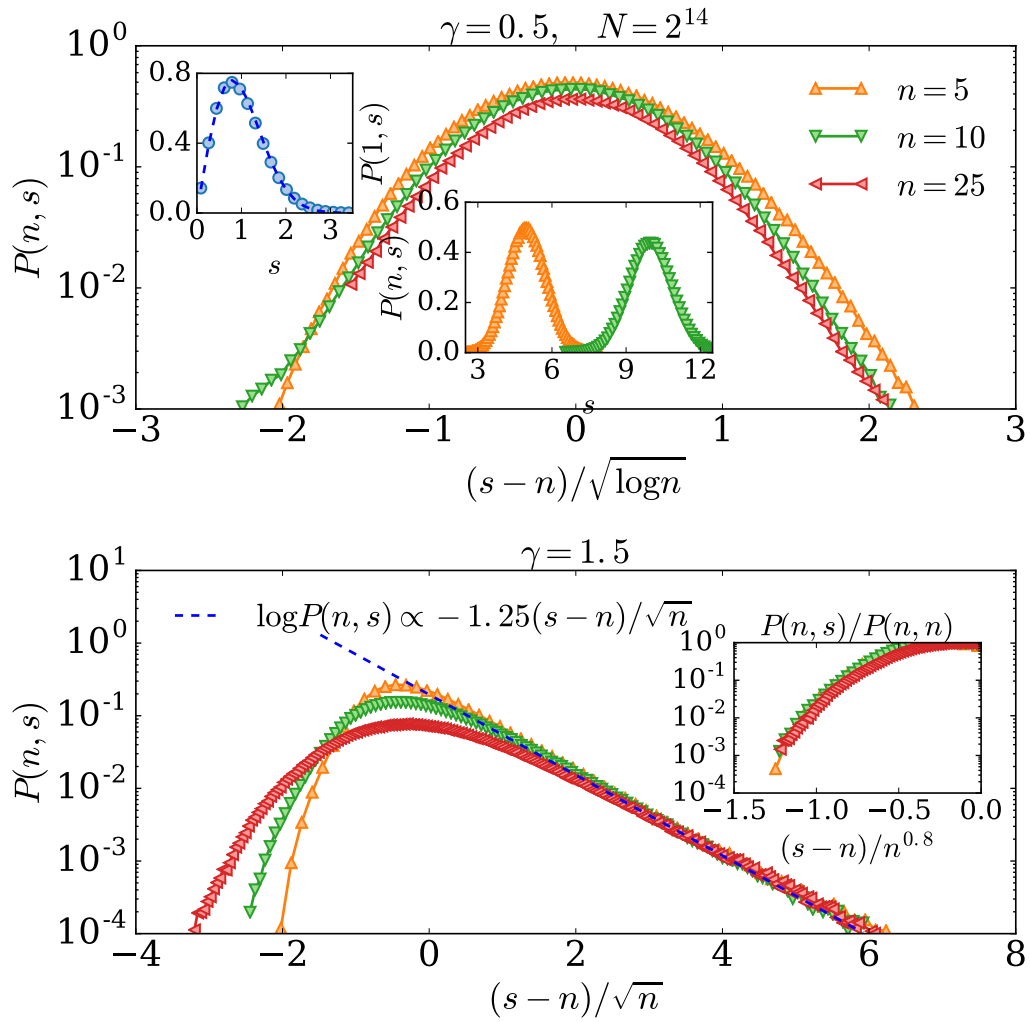

Figure 4: ((Color online) (upper panel) Gaussian $\ln P(s, n) \sim-(s-n)^{2} / \operatorname{var}(n)$ in the ergodic phase $\gamma=0.5$. The variance $\operatorname{var}(n) \sim \ln (n)$ as it should be for the Wigner-Dyson statistics. (lower panel): $P(s, n)$ in the NEE phase $\gamma=1.5$. The behavior of $P(s, n)$ for $s>n$ is quasi-

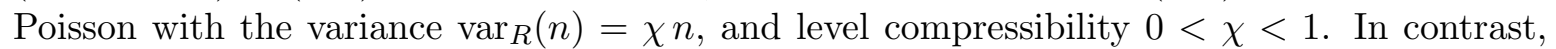
for $s<n$ the function $\ln P(s, n)$ is non-linear in $s$ with the variance $\operatorname{var}_{L}(n) \sim n^{1.6}$. Such a super-Poisson behavior reflects the mini-band structure of spectrum with level correlations inside mini-bands much stronger than between of them.

\section{Numerical test for ergodicity}

Fig. 3 shows $R(t)$ obtained by numerical diagonalization of GRP matrix Hamiltonians of the size $N$ up to $N=2^{14} 7$. The results, which should be compared with Fig. 2, are quantitatively described by the model of the rigid system of energy levels discussed in the previous sections, (29), see the theoretical dashed curve in Fig. 3.

The oscillations in $R(t)$ can be used as the numerical test for fully ergodic wave functions not only in GRP but also in other models $23,25,26,36$, including many-body quantum systems 8 .

\footnotetext{
${ }^{7}$ The numerical data shown in the paper is averaged over $N_{r}=1000$ disorder realizations and $N_{x}=16$ basis states $\left|\Psi_{0}\right\rangle$.

${ }^{8}$ However, in some numerical works (see, e.g., 27]), the oscillations of the survival probability in a manybody interacting spin system survive even in the localized phase.
} 


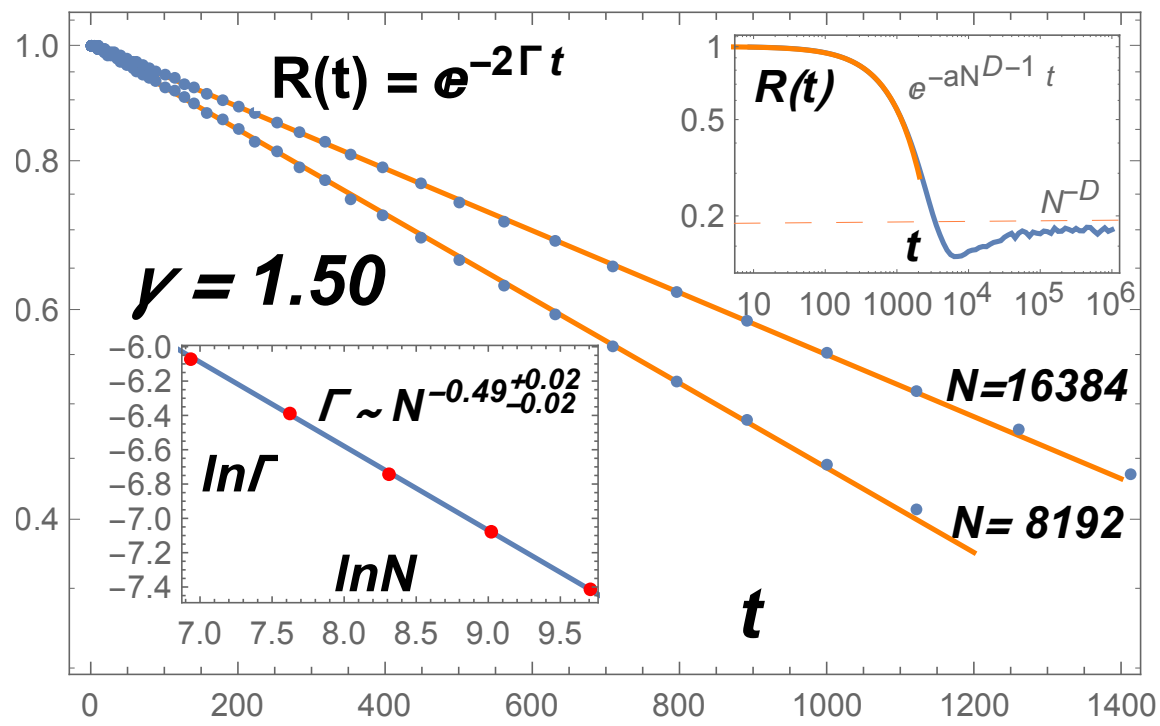

Figure 5: (Color online) Exponential decay of survival probability in NEE phase of GRP model with $\gamma=1.5, \lambda=0.1$. (upper inset) Global view of survival probability including the asymptotic time-independent regime. (lower inset) Extraction of $D \approx 0.49 \pm 0.02$ at $\gamma=1.5$ from the decay rate $2 \Gamma(N) \propto N^{-1+D}$.

\section{Extracting fractal dimension $D$ from $R(t)$ in the NEE phase}

As it follows from Eq. (26) the parameter $c \propto N^{1-D}$ in Eq. (27) which determines $R(t)$ via Eq. (25), is infinite in the thermodynamic limit $N \rightarrow \infty$ in the NEE phase characterized by $D<1$. In this case $R(t)-R(\infty)$ coincides with Eq. (23) in the thermodynamic limit $N \rightarrow \infty$. Then the scaling of the mini-band width, Eq. (9), gives access to the fractal dimension $D$ through measuring the decrement $2 \Gamma$ of the exponential decay. In Fig. 5 (which should be compared with Fig. 2(b)) we present the plots of $R(t)$ obtained by exact diagonalization of matrix Hamiltonian from GRP ensemble and show an example of extraction of $D(\gamma)$ at $\gamma=1.5$.

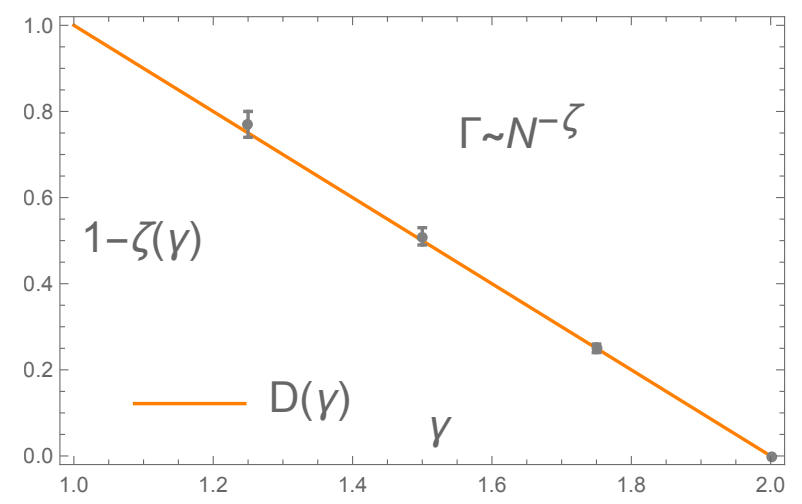

Figure 6: (Color online) Fractal dimension $D(\gamma)=1-\zeta$ extracted numerically from $\Gamma(N) \propto$ $N^{-\zeta}$ using Eq. (9) and the theoretical prediction $D=2-\gamma$ of Ref. [15 (solid line). 

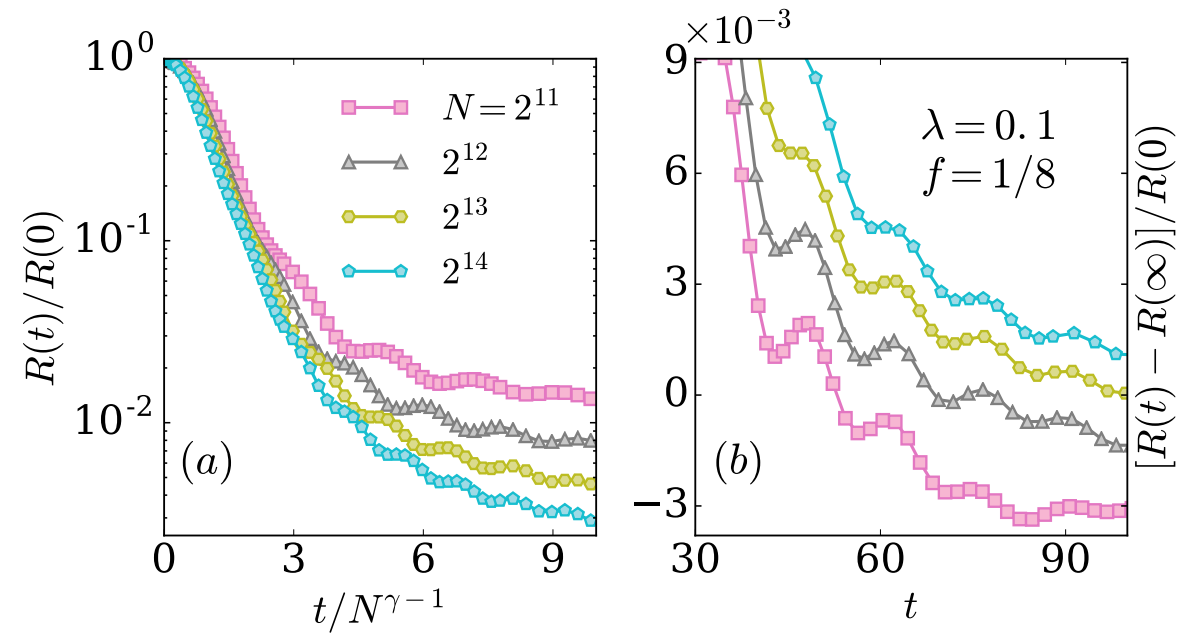

Figure 7: (Color online) Residual oscillations in $R(t)$ in the NEE phase with $\gamma=1.25$ and $\lambda=1$ versus (a) rescaled time collapsing exponential decay, (b) unscaled time. Oscillations decay with the increasing system size and the exponential behavior Eq. (23) emerges. Period of oscillations does not scale with the system size in accordance with Eq. (28).

Fig. 6 demonstrates that this method of extraction of $D$ from the exponential decay $\Gamma$ scaling with $N$ as $\Gamma(N) \propto N^{-\zeta}$, Eq. (9), is very accurate, shows $\zeta=1-D=\gamma-1$, and does not suffer from large finite-size corrections. It should be noted that the alternative method of extraction of $D$ from the asymptotic value $R(\infty)$ of $R(t)$ (shown by a dashed line in upper inset in Fig. 5 requires much larger system sizes and much more computational efforts.

\section{Residual oscillations in $R(t)$ in the NEE phase}

One should also take care of the residual oscillations in $R(t)$ with the same universal period, Eq. (28), which are present at a finite $N$ in the NEE phase close to ergodic transition at $\gamma=1$ when $c \propto \lambda^{-2} N^{\gamma-1}$ is not large enough. They can be suppressed either by increasing the system size or by decreasing a value of the constant $\lambda$ in Eq. (2), cf. Figs. 5 and 7.

In Fig. 7 we demonstrate how with increasing the system size in the NEE phase, $\gamma>1$, the oscillations die out giving way to the exponential decay. We also demonstrate that the exponential decay has a different scaling with $N$ than the oscillating part, the period of oscillations being $N$-independent.

\section{$11 R(t)$ in the localized phase}

In Fig. 1 we give an overview of the evolution of the form of $R(t)$ with increasing $\gamma$ in all three phases. The localization transition at $\gamma=2$ is marked by the scale-independent $R(t)$ that has a finite limit $R(\infty) \propto I_{2}$ at $N \rightarrow \infty$. In the localized phase $\gamma>2$ the limiting value $R(\infty)$ remains finite.

For finite $N$ the asymptotic value $R(\infty)$ depends on the constant $\lambda$ in Eq. (2), Fig. 8 , 

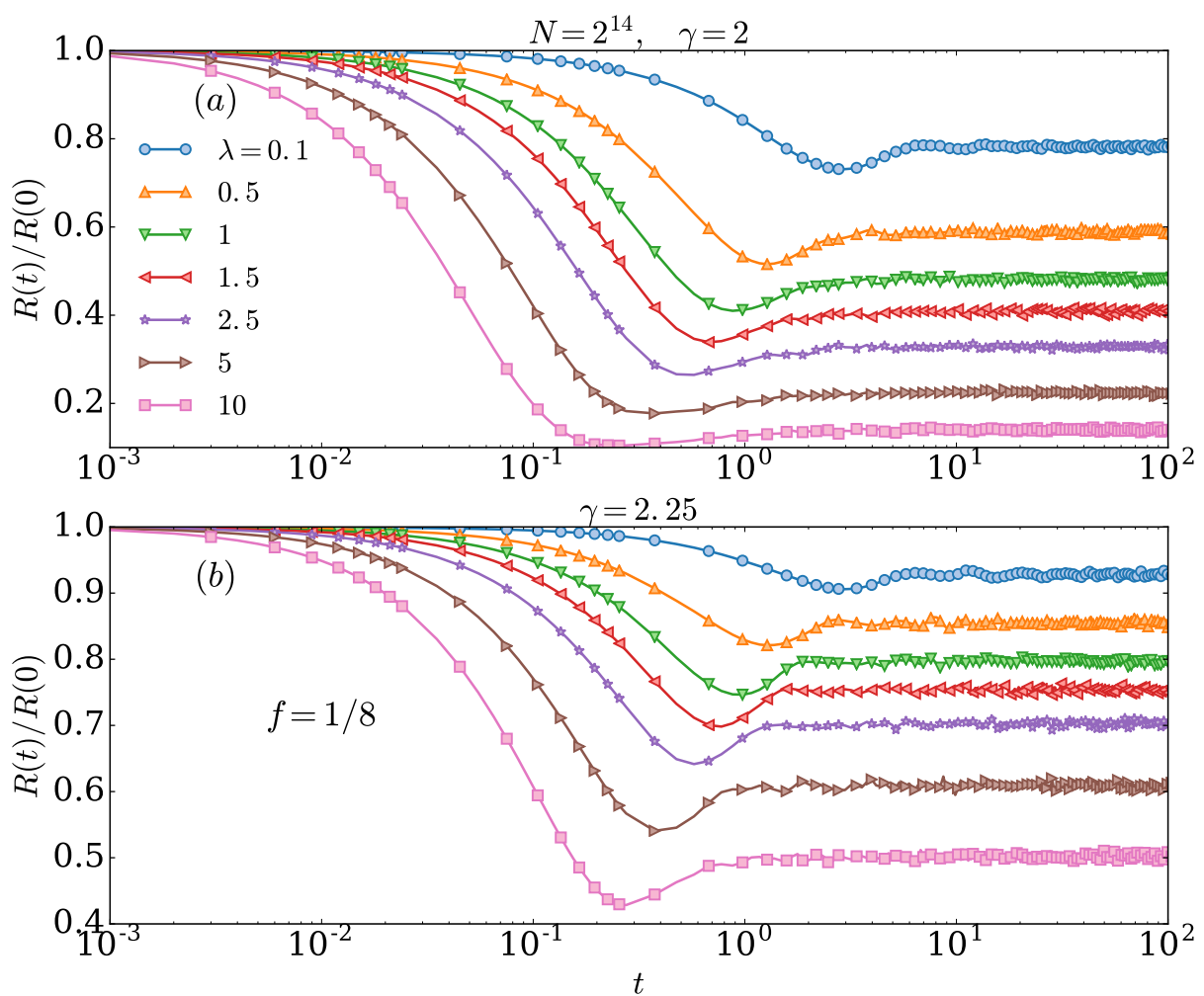

Figure 8: (Color online) Finite- $N$ survival probability $R(t)$ (a) at the critical point of localization transition $\gamma_{c}=2$ and (b) in the insulating phase $\gamma=2.25$ for different values of $\lambda$ in Eq. (2). The parameters are $N=2^{14}$ and $f=1 / 8$.

At a fixed $\lambda$ and increasing $N$ the value $R(\infty)$ stays constant in the critical point $\gamma_{c}=2$ of the localization transition but it moves towards its initial value $R(0)$ in the insulating phase (see Fig. 9 and Fig. 10). This is related with the special property 15 of the localized phase in GRP ensemble that there is a gap between the peak value, Eq. (13), of $\left|\psi_{n}(n)\right|^{2} \approx 1$ and the typical maximal value of $\left|\psi_{n}(r \neq n)\right|^{2} \sim N^{-(\gamma-2)} \ll 1$ (see Eq. (8) and 18 for details). This implies that the Lyapunov exponent is divergent in the thermodynamic limit, as for the Bethe lattice with infinite connectivity [37.

\section{Conclusions}

In summary, the detailed analysis of the survival probability $R(t)$ defined in Eq. (4) in the generalized Rosenzweig-Porter ensemble [15] demonstrates three distinctly different types of behavior in different phases. The ergodic phase shows robust oscillations in $R(t)$ with a standard polynomial decay $\sim t^{-2}$ of their amplitude with time and a universal frequency equal to the bandwidth of the initially prepared wavepacket. These oscillations essentially depend on the initial spectral decomposition of the prepared wave packet and in the case of the projected 


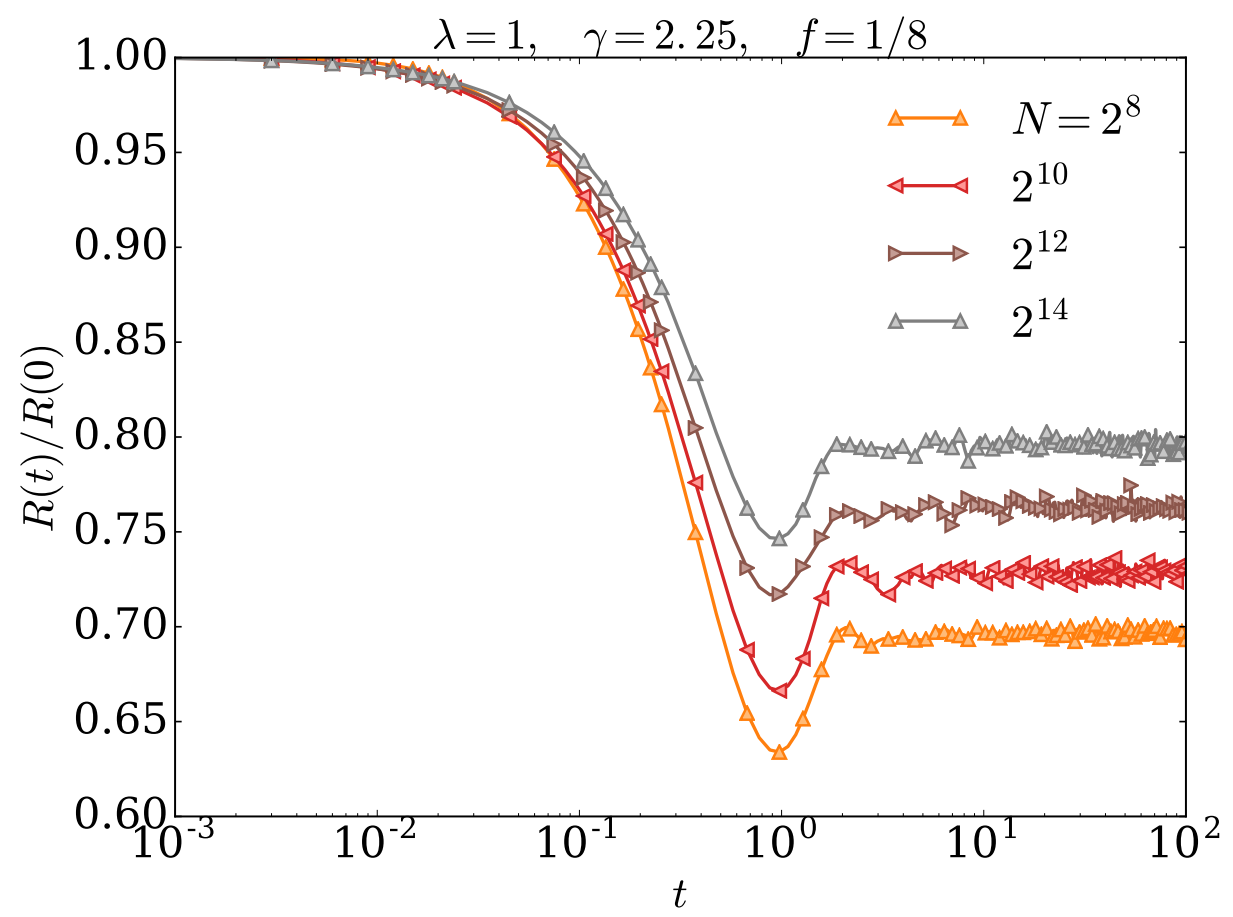

Figure 9: (Color online) Evolution of $R(t)$ in the insulating phase at $\gamma=2.25$ with $\lambda=1$ and $f=1 / 8$. With increasing $N$ the asymptotic value $R(\infty)$ moves towards its initial value $R(0)$.

basis state, Eq. (4), provide a probe of ergodicity of the extended states. In the multifractal phase no oscillations persist in the thermodynamic limit and the survival probability decays exponentially, albeit with a small rate which is related to the fractal dimension of the eigenstates. An exponential decay of the survival probability in this phase is nearly free of the finite size effects and provides an accurate way to extract the multifractal dimension of eigenstates from the decay rate. Localized phase is characterized by the universal size-independent saturation value $R(t \rightarrow \infty)=R(\infty)$ coinciding with the initial value $R(t \rightarrow 0)=R(0)$ in the thermodynamic limit $N \rightarrow \infty$.

\section{Acknowledgements}

We are grateful to L. B. Ioffe and M. V. Feigel'man for stimulating discussions on the applicability of this model to Quantum Random Energy Problem. IMK acknowledges the support of the Russian Foundation for Basic Research and German Research Foundation (DFG) Grant No. KH 425/1-1. SB acknowledges support from DST, India, through Ramanujan Fellowship Grant No. SB/S2/RJN-128/2016. VEK and SB acknowledge hospitality of the Max-Planck Institute for the Physics of Complex Systems, Dresden, Germany. MA acknowledges hospitality of the International Centre for Theoretical Physics, Trieste, Italy. VEK acknowledges hospitality of KITP at UCSB where the final part of the work was done and the support from the National Science Foundation under Grant No. NSF PHY-1748958. 

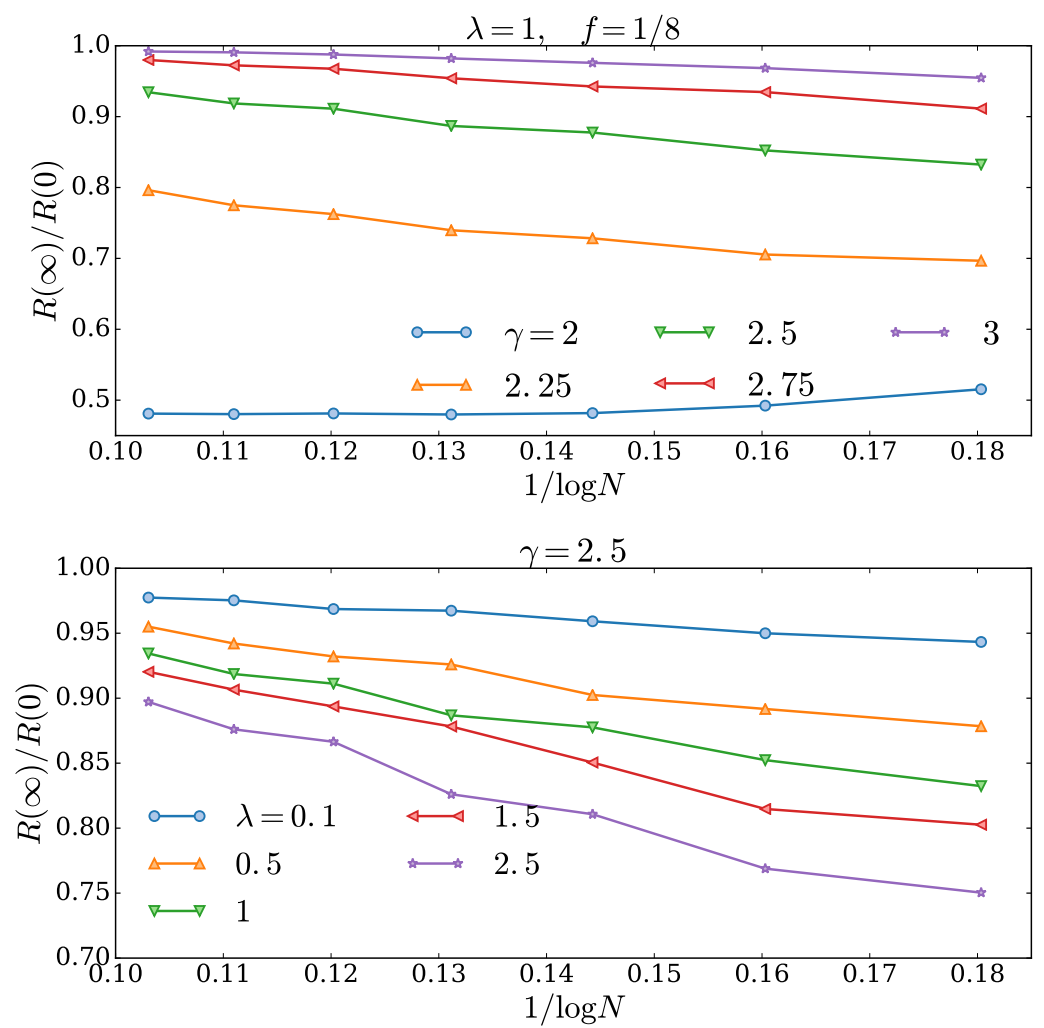

Figure 10: (Color online) The ratio $R(\infty) / R(0)$ as a function of $1 / \ln N$ (upper) for different values of $\gamma$ at $\lambda=1$ and $f=1 / 8$ and (lower) for different values of $\lambda$ at $\gamma=2.5$. For all $\gamma>2$ in the insulating phase $R(\infty) / R(0)$ increases with increasing the system size and should approach 1 in the thermodynamic limit. At the localization transition $\gamma_{c}=2$ the ratio $R(\infty) / R(0)$ is saturated for $N>10000$ at a $\lambda$-dependent value smaller than 1 .

\section{References}

[1] D. M. Basko, I. L. Aleiner, B. L. Altshuler. Ann. Physics 321, 112 (2006).

[2] I. V. Gornyi, A. D. Mirlin, and D. G. Polyakov, Phys. Rev. Lett. 95, 206603 (2005).

[3] V. Oganesyan and D. A. Huse, Phys. Rev. B, 75, 155111 (2007).

[4] D. J. Luitz, Y. Bar Lev, Phys. Rev. Lett. 117, 170404 (2016).

[5] M. Žnidarič, A. Scardicchio, V. K. Varma, Phys. Rev. Lett. 117, 040601 (2016).

[6] D. J. Luitz, N. Laflorencie, and F. Alet, Phys. Rev. B 93, 060201(R) (2016).

[7] M. Serbyn, Z. Papic, D. A. Abanin Phys. Rev. B 96, 104201 (2017).

[8] M. Pino, L. B. Ioffe, and B. L. Altshuler, PNAS, 113, 536 (2016).

[9] M. Pino, V. E. Kravtsov, B. L. Altshuler, and L. B. Ioffe, Phys. Rev. B 96, 214205 (2017). 
[10] F. Evers and A. D. Mirlin, Rev. Mod. Phys. 80, 1355 (2008).

[11] V. N. Smelyanskiy, K. Kechedzhi, S. Boixo, S. V. Isakov, H. Neven, B. L. Altshuler, arXiv:1802.09542 (2018).

[12] S. Bera, G. De Tomasi, F. Weiner, F. Evers, Phys. Rev. Lett. 118, 196801 (2017).

[13] R. Steinigeweg, J. Herbrych, F. Pollmann, and W. Brenig, Phys. Rev. B 94, 180401(R) (2016).

[14] N. Rosenzweig and C. E. Porter, Phys. Rev. B 120, 1698 (1960).

[15] V. E. Kravtsov, I. M. Khaymovich, E. Cuevas, M. Amini, New J. Phys. 17, 122002 (2015).

[16] P. von Soosten, S. Warzel, arXiv:1709.10313 (2017).

[17] P. A. Nosov, I. M. Khaymovich, and V. E. Kravtsov, arXiv:1810.01492 (2018).

[18] P. A. Nosov and I. M. Khaymovich, in preparation.

[19] S. Roy, I. M. Khaymovich, A. Das, and R. Moessner, SciPost Phys. 4, 025 (2018).

[20] D. Facoetti, P. Vivo and G. Biroli, EPL 11547003 (2016).

[21] C. Monthus, J. Phys. A: Math. Theor. 50, 295101 (2017).

[22] M. L. Mehta, Random matrices, Elsevier, (2004).

[23] M. Távora, E. J. Torres-Herrera, and L. F. Santos, Phys. Rev. A 95, 013604 (2017).

[24] F.Borgonovi, F.M.Izrailev, L.F.Santos, V.G.Zelevinsky, Phys. Rep. 626, 1-58 (2016)

[25] E. J. Torres-Herrera, A. M. García-García, and L. F. Santos, Phys. Rev. B 97, 060303(R) (2018).

[26] L. F. Santos and E. J. Torres-Herrera, arXiv:1803.06012 (2018).

[27] E. J. Torres-Herrera and L. F. Santos, Phys. Rev. B 92, 014208 (2015).

[28] E. Bogomolny and M. Sieber, Phys. Rev. E 98, 032139 (2018).

[29] E. Cuevas and V. E. Kravtsov, Phys. Rev. B. 76, 235119 (2007).

[30] T. Guhr, Annals of Physics 250, 145 (1996).

[31] Y. V. Fyodorov and A. D. Mirlin, Phys. Rev. B 55, 16001 (1997).

[32] M. V. Feigel'man, L. B. Ioffe, V. E. Kravtsov, E. A. Yuzbashyan, Phys. Rev. Lett. 98, 027001 (2007).

[33] M. V. Feigel'man, L. B. Ioffe, V. E. Kravtsov, E. Cuevas, Ann. Phys. 325, 1390 (2010).

[34] M. V. Feigel'man and V. E. Kravtsov, arxiv:1809.09888. 
[35] B. I. Shklovskii, B. Shapiro, B. R. Sears, P. Lambrianides, and H. B. Shore, Phys. Rev. B 47, 11487 (1993).

[36] G. de Tomasi, S. Bera, I. M. Khaymovich, and A. Scardicchio, Phys. Rev. B 98, 134205 (2018).

[37] V. E. Kravtsov, B. L. Altshuler and L. B. Ioffe, Annals of Physics, 389, 148 (2018).

\section{Appendix}

Eq. (24) can be evaluated using the Poisson summation formula:

$$
\sum_{\ell=-\infty}^{+\infty} F(\ell)=\sum_{m=-\infty}^{+\infty} \int_{-\infty}^{+\infty} e^{2 \pi i m x} F(x) d x .
$$

For delocalized states, the function $F(x)$ takes the form:

$$
F(x)=A\left(1-\frac{|x|}{f N}\right) \frac{\cos (x t \delta)}{x^{2}+g^{2}} \theta(f N-|x|),
$$

where $A=2 \pi f \bar{\rho}(0)^{2} \lambda^{4} N^{2(1-\gamma)} /(\Gamma \delta), \bar{\rho}(0)$ is the mean DoS and $g=2 \Gamma / \delta$.

Evaluating the integral:

$$
\begin{aligned}
& Y(k, c)=\int_{-c}^{c}(1-|y| / c) \frac{\cos (2 \pi k y)}{1+y^{2}} d y \\
& =\cosh (2 \pi|k|)[\pi+\dot{\mathrm{i}} \operatorname{ci}(2 \pi|k|(c+\mathrm{i}))-\dot{\mathrm{i}} \operatorname{ci}(2 \pi|k|(c-\mathrm{i}))] \\
& -\sinh (2 \pi|k|)[\operatorname{si}(2 \pi|k|(c+\mathrm{i}))+\operatorname{si}(2 \pi|k|(c-\mathrm{i}))] \\
& +\frac{1}{c} \cosh (2 \pi|k|)[2 \operatorname{ci}(2 \pi|k|)-\operatorname{ci}(2 \pi|k|(c+\mathrm{i}))-\operatorname{ci}(2 \pi|k|(c-\mathrm{i}))]- \\
& -\frac{1}{c} \sinh (2 \pi|k|)[2 \operatorname{si}(2 \pi|k|)+\dot{\mathrm{i}} \operatorname{si}(2 \pi|k|(c+\mathrm{i}))-\mathrm{i} \operatorname{si}(2 \pi|k|(c-\dot{\mathrm{i}}))] .
\end{aligned}
$$

with $y=x \delta t /(2 \pi k), k=k_{m, \pm}=\frac{t \Gamma}{\pi} \pm 2(\Gamma / \delta) m$, and $c=\frac{f N \delta}{2 \Gamma}$, we can express the r.h.s. of Eq. (35) in terms of the cosine integral $(\mathrm{ci}(\mathrm{x}))$ and the sine integral $(\mathrm{si}(\mathrm{x}))$ functions of complex arguments $c \pm \dot{i}$. The first two lines in Eq. (37) correspond to the first term in $(1-|x| / c)$, and the last two lines follow from the second term $-|x| / c$. At large $k$, the leading oscillating terms $\propto \sin (2 \pi c|k|) /|k|$ in these two contributions cancel out. 